\title{
Neonatal pain response to heel stick vs venepuncture for routine blood sampling
}

\author{
Vibhuti S Shah, Anna Taddio, Sian Bennett, Brian D Speidel
}

\begin{abstract}
Neonatal pain response and adverse effects and maternal anxiety were assessed in 27 infants who were randomly allocated to venepuncture or heel stick. Pain was assessed by nurses using the Neonatal Infant Pain Scale (NIPS) and a three point scale for the mothers. NIPS scores were higher in the heel stick group compared with the venepuncture group. Maternal anxiety was higher before the procedure while perception of an infant's pain was lower in the venepuncture group compared with the heel stick group. Venepuncture is less painful than heel stick in newborn infants undergoing routine blood sampling.

(Arch Dis Child 1997;77:F143-F144)
\end{abstract}

Keywords: pain response; heel stick; venepuncture; blood sampling

Heel stick is the conventional method of blood sampling in neonates. Previous research has shown that infants undergoing heel stick show signs of distress and pain. ${ }^{12}$ There are currently no published data comparing pain response with an alternative method of blood sampling such as venepuncture.

Venepuncture is a common procedure performed in older infants and children. ${ }^{3}$ Its advantages include increased sample volume, reduced risk of a haemolysed or clotted sample, and perhaps less pain. The benefit of heel stick is that it is very easy to perform, and therefore paramedics can also do the task.

The primary objective of the study was to compare the pain response to different methods of blood sampling in full term infants. The secondary objectives were to compare the incidence of adverse effects.

Neonatal Intensive Care Unit, Southmead Hospital, Bristol

V S Shah

A Taddio

S Bennett

B D Speidel

Correspondence to: Dr Vibhuti S Shah, Shared Program in Neonatology,

University of Toronto,

The Hospital for Sick Children,

555 University Avenue,

Toronto, Ontario,

M5G 1X8, Canada.

Accepted 1 May 1997

\section{Methods}

The study was approved by the research ethics committee and informed parental consent was obtained. Healthy neonates of $>37$ weeks gestational age having blood taken for measurement of serum bilirubin or glucose were eligible. The babies were randomly assigned to the heel stick or venepuncture sampling technique. Randomisation was computer generated. One investigator (VS) performed all procedures. Heel stick and venepuncture were performed in a standardised manner while the infant was held in the mother's or nurse's arms. For the heel stick group, the heel was wiped with alcohol, pricked with a lancet and squeezed to collect the required volume of blood $(0.25 \mathrm{ml})$. A cotton wool ball was applied to prevent bleeding. For venepuncture, the investigator identified the site (most common site used was the dorsum of the hand), cleaned it with alcohol, punctured the vein using a 21 gauge needle and collected $0.25 \mathrm{ml}$ of blood. A cotton wool ball was applied to prevent bleeding after the needle was removed. The mother and the nurse responsible for care of the baby were present during the procedure. The nurse rated the infant's pain using the Neonatal Infant Pain Scale(NIPS). ${ }^{4}$ The total score ranges from 0 to 7 . Pain assessments were made at baseline (before the procedure), during (when maximal response was observed), and after the procedure (when cotton wool was applied). Nurses were blinded to the purpose of the study.

At the end of the procedure, each mother completed a questionnaire rating her anxiety level before the procedure using a three point scale: $0=$ not worried at all, $1=$ a little worried and $2=$ very worried. Mothers also rated their babies' pain response using a three point scale: $0=$ no pain at all, $1=$ a little pain, and $2=$ a lot of pain. The number, reasons for multiple attempts, and occurrence of adverse effects were noted.

To achieve a difference in the NIPS score of 2 between each group, with $80 \%$ power and $\mathrm{P}$ value of $<0.05$, we estimated a sample size of 25 babies in each group. Due to unforeseen circumstances unrelated to the study, an analysis was performed on 27 subjects which revealed significant differences between groups. Consequently, the study was prematurely stopped.

NIPS and maternal pain ratings were compared between groups using Student's $t$ test. Demographic characteristics were compared using Fisher's exact test, or Student's $t$ test, as appropriate. A P value of $<0.05$ was considered significant.

\section{Results}

Twenty seven neonates were enrolled, 14 of whom were randomly allocated to heel stick (group 1) and 13 to venepuncture (group 2). The demographic details of the babies are shown in table 1 . No significant differences were observed between treatment groups. One baby in each group had the procedure performed while being breast fed.

The NIPS scores before and after the procedure did not differ between groups. During the procedure, NIPS scores were significantly higher in group $1(\mathrm{P}<0.001)$ (fig 1$)$. Although 
Table 1 Demographic characteristics of study subjects *

\begin{tabular}{lccl}
\hline Variable & Heel stick & Venepuncture & Pvalue \\
\hline Postnatal age (days) & $3.1(1.1)$ & $2.8(1.2)$ & 0.61 \\
Gender (\% male) & $8(57.2 \%)$ & $8(61.5 \%)$ & 0.82 \\
Reason for test (\% bilirubin) & $13(93 \%)$ & $12(97 \%)$ & 0.96 \\
Time of last feed (hours) & $1.8(1.5)$ & $1.6(1.6)$ & 0.79 \\
State (\% awake before procedure) & $5(35.5 \%)$ & $7(58.3 \%)$ & 0.25 \\
\hline
\end{tabular}

^Values are mean (SD) or number (\%).

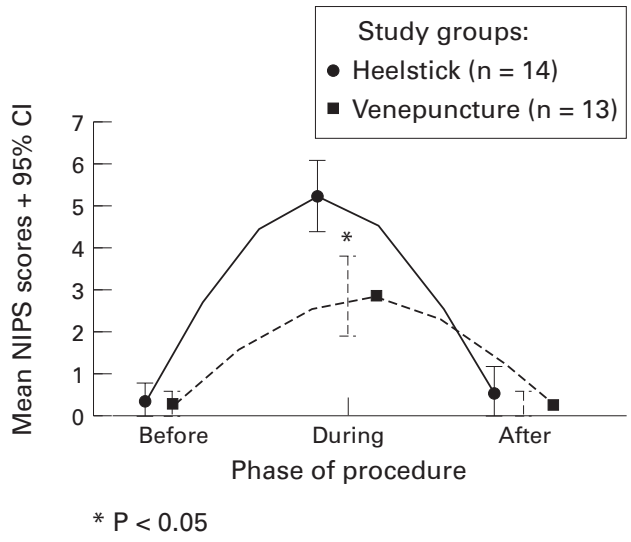

Figure 1 Comparison of heel stick and venepuncture scores in 27 infants.

the mother's anxiety state beforehand was higher for group $2(2.2+0.7)$ vs group 1 (1.4 $+0.5)(\mathrm{P}<0.001)$. Mothers of infants who received venepuncture rated their infants' pain levels lower $(1.6+0.5)$ than those whose infants received heel stick $(2.4+0.5)(\mathrm{P}<0.001)$. Four neonates in group 1 and one neonate in group 2 required a second prick to obtain adequate sample volume $(\mathrm{P}=0.33)$. Two of the four $(50 \%)$ neonates with multiple heel sticks had haemolysed samples that required venepuncture. One neonate in group 1 had bruising at the local site.

\section{Discussion}

Heel stick is a common method of blood collection in neonates. Various pharmacological and non-pharmacological manoeuvres have been used in an effort to reduce pain and distress during heel stick, but no previous study has compared the pain response and acceptability by parents of heel stick with other blood sampling methods, such as venepuncture. This study revealed that neonates who underwent a heel stick had higher pain scores than those undergoing venepuncture and on some occasions required repeated sampling. Although the mothers were more worried about the venepuncture, after observing the procedure, their rating suggested that heel stick was more painful than venepuncture.

The management of pain in the newborn is a topical and important issue. Until recently, it was believed that infants and young children could not feel pain due to the immaturity of their nervous system. Previous studies have clearly shown that the anatomical, physiologi$\mathrm{cal}$, and neurochemical structures which convey pain are well developed in both preterm and term neonates. ${ }^{5}$ Another common belief was that pain could not be accurately assessed in this age group. However, there are now many validated tools for measuring pain in the newborn-for example, NIPS ${ }^{4}$ and the Neonatal Facial Coding System (NFCS). ${ }^{6}$ Therefore, it is appropriate that clinicians attempt to reduce the number of harmful components involved in the care of the neonates. We have shown that venepuncture is associated with less pain than heel stick, and recommend that venepuncture should be preferentially used in term neonates for blood sampling. Currently, venepuncture is normally performed by medical staff and more widespread use of venepuncture will require paramedical staff to be appropriately trained.

1 Brown L. Physiologic responses to cutaneous pain in neonates. Neonatal Network 1987;5:18-21.

2 Owens ME,Todt EH. Pain in infancy: neonatal reaction to heel lance. Pain 1984;20:77-86.

3 McKay RJ. Diagnosis and treatment: risks of obtaining samples of venous blood in infants. Pediatrics 1966;38:906-8. 4 Lawrence J, Alcock D, Mc Grath P, Kay J, et al. The development of a tool to assess neonatal pain. Neonatal Network 1993;12:59-66.

5 Fitzgerald M, McIntosh N. Pain and analgesia in the newborn. Arch Dis Child 1989;64:441-3.

6 Grunau RVE, Craig KD. Pain expression in neonates: Facial action and cry. Pain 1987;28:395-410. 